\title{
BMJ Open Quality Enhancing teamwork communication and patient safety responsiveness in a paediatric intensive care unit using the daily safety huddle tool
}

\author{
Fatima Aldawood (D) , ' Yasser Kazzaz (D) , 2,3,4 Ali AlShehri, ${ }^{2,3,4}$ Hamza Alali, ${ }^{3,4}$ \\ Khaled Al-Surimi ${ }^{5,6}$
}

To cite: Aldawood F, Kazzaz Y, AlShehri A, et al. Enhancing teamwork communication and patient safety responsiveness in a paediatric intensive care unit using the daily safety huddle tool. BMJ Open Quality 2020;9:e000753. doi:10.1136/ bmjoq-2019-000753

Received 15 June 2019 Revised 23 January 2020 Accepted 11 February 2020

D Check for updates

(C) Author(s) (or their employer(s)) 2020. Re-use permitted under CC BY-NC. No commercial re-use. See rights and permissions. Published by BMJ.

${ }^{1}$ Nursing Services, Ministry of National Guard Health Affairs, Riyadh, Central, Saudi Arabia ${ }^{2}$ College of Medicine, King Saud bin Abdulaziz University for Health Sciences, Riyadh, Saudi Arabia

${ }^{3}$ King Abdullah International Medical Research Center,

Riyadh, Saudi Arabia

${ }^{4}$ Department of Pediatric, King Abdulaziz Medical City, Riyadh, Saudi Arabia

${ }^{5}$ College of Public Health and Health Informatics, King Saud bin Abdulaziz University for Health Sciences, Riyadh, Saudi Arabia

${ }^{6}$ Primary Care and Public Health Department, School of Public Health, Imperial College London, London, UK

Correspondence to

Ms Fatima Aldawood;

dawood.fatima@outlook.com

\section{ABSTRACT}

Background Open communication between leadership and frontline staff at the unit level is vital in promoting safe hospital culture. Our hospital staff culture survey identified the failure to address safety issues as one of the areas where staff felt unable to express their concerns openly. Thus, this improvement project using the daily safety huddle tool has been developed to enhance teamwork communication and respond effectively to patient safety issues identified in a paediatric intensive care unit. Methods We used the TeamSTEPPS quality approach. TeamSTEPPS is an evidence-based set of teamwork tools developed by the US Agency of Healthcare Research and Quality to enhance teamwork and communication. We applied TeamSTEPPS using a tool called the Daily Safety Huddle, aiming at improving communication and interaction between healthcare workers and building trust by acting immediately when there is any patient safety issue or concern at the unit level.

Results During the period from April to December 2017, the interaction between frontline staff and unit leadership increased through compliance with the daily safety huddle. Initially, compliance was at $73 \%$, but it increased to $97 \%$, with a total of 340 safety issues addressed. The majority of these safety issues pertained to infection control and medication errors (109; 32.05\%), followed by communication $(83 ; 24.41 \%)$, documentation $(59$; $17.35 \%)$, other issues $(37 ; 10.88 \%)$, procedure $(20$; $5.88 \%)$, patient flow $(16 ; 4.7 \%)$ and equipment and supplies $(16 ; 4.7 \%)$.

Conclusions Systematic use of daily safety huddle is a powerful tool to create an equitable environment where frontline staff can speak up freely about daily patient safety concerns. The huddle leads to a more open and active discussion with unit leadership and to the ability to perform the right action at the right time.

\section{INTRODUCTION}

\section{Problem assessment}

In 2014, MNGHA (Ministry of National Guard - Health Affairs) conducted an institutionwide culture survey. The results from the paediatric intensive care unit (PICU) showed weakness in the domain of safety climate. The majority of staff claimed that they did not receive proper feedback about their performance, and they expressed fear of and difficulty in discussing errors. Moreover, some staff felt the culture of the unit did not promote learning from errors. In order to better understand the problem and select an appropriate intervention, the quality and patient safety department conducted a meeting involving frontline staff and unit leadership (division head, unit-based quality steering committee, nurse manager, clinical resource nurses and nurse coordinators). The meeting revealed the need for transparency in order for staff to address and report safety issues. In addition, leaders acknowledged the need for dedicated time with staff to develop trust and provide constant feedback. Staff and leaders realised that errors could be due to human factors like poor teamwork and poor communication rather than individual mistakes.

\section{Background}

Open communication between leadership and staff at the unit level is vital in promoting safe hospital culture. ${ }^{1}$ Daily quick meetings are proven to improve a team's dynamics through sharing knowledge and discussing safety issues. ${ }^{2}$ Moreover, a timely leadership response to frontline staff's concerns builds trust and brings the team together, which is positively reflected in the care provided to patients and their families. ${ }^{2}$ Therefore, the US Institute for Healthcare Improvement (IHI) and the UK National Health Service (NHS) recommend the use of the daily safety huddle in clinical settings. ${ }^{34}$

Conversations that take place in the huddle between individuals who might not otherwise interact may help to combat the perpetuation of unconstructive communication norms. For example, staff working in highreliability organisations should be aware of what is known as 'the fallacy of centrality: the 
assumption that someone in a central or senior position will be aware of, or informed about any significant occurrences'. ${ }^{5}$ Although personnel are generally more likely to mistakenly believe this and to not communicate information, leaders might also, unintentionally, take part in this fallacy. ${ }^{5}$ Regular huddles enhance relationships because care providers get to know each other and can help each other overcome cultural taboos that have prevented previous discussions. ${ }^{5}$

Engaging frontline staff with leadership to address and discuss safety issues plays a major role in improving both the quality of care delivered to patients and teamwork; this approach increases staff awareness and builds trust and commitment to the decisions made. Failure to openly address safety issues and errors could lead to an accumulation of clinical issues with detrimental consequences for paediatric critical care patients, making them a special area of concern. ${ }^{6}$ Complex settings like our paediatric critical care unit, which consists of multiple teams, require a high level of coordination and interprofessional communication.

Previous success stories of team building and coordination provided encouragement as we planned improvements in our unit. One paediatric radiology department implemented the daily safety huddle, ${ }^{7}$ and another study in the UK showed the positive impact of huddles in a sample of National Health Service (NHS) paediatric wards where the Situation Awareness For Everyone (SAFE) programme was implemented. The huddle was a central component of the programme. ${ }^{8}$

Our journey of improvement took place in the Paediatric ICU- King Abdullah Specialised Children Hospital (KASCH), King Abdulaziz Medical City, Riyadh, Kingdom of Saudi Arabia, which is composed of 20 beds serving critically ill paediatric patients in a patient-family centred care environment. The multidisciplinary team comprised physicians, nurses, respiratory therapists, a dietician, a clinical pharmacist, a physiotherapist, an occupational therapist and others. Because the unit care team involves many disciplines, clear communication is required to ensure care is aligned and carried out as planned. We wanted to create a learning culture in which the team shares knowledge, experience and mutual support.

\section{METHODS}

\section{Tool selection}

TeamSTEPPS is an evidence-based set of teamwork tools developed by the US Agency of Healthcare Research and Quality with the goal of optimising patient outcomes by improving communication and teamwork skills. ${ }^{9}$ The quality and patient safety department of our hospital nominated staff to receive a master training course held locally following many safety incidents resulted because of poor teamwork and miscommunication. Following the master training course, the quality and patient safety department selected many units to attend. Our unit leadership and frontline staff attended the TeamSTEPPS workshop. The staff that attended the workshop selected two recommended tools for implementation, one being the daily safety huddle. Based on the findings of the hospital culture survey, we selected this tool to transform the relationship between the leadership and frontline staff through creating time and space for meaningful conversations to enhance relationships and strengthen a culture of safety. Huddles can be of particular value to healthcare organisations seeking or sustaining reliability. ${ }^{5}$

\section{Planning}

The team consisted of the division head of the unit, a physician and a clinical resource nurse under the supervision of the PICU's quality steering committee. We customtailored the structure of the daily huddle after exploring how daily huddles are conducted in other institutions. We planned the huddle presuming that it would not be easy to gather all the staff due to the horseshoe-shaped design of the unit and the nature of our patients; the majority are critically ill and require close monitoring and continuous primary healthcare provider attendance. For that reason, we agreed that the huddle would be conducted in our unit on a daily basis at a fixed place at 1400 hours for a maximum of $15 \mathrm{~min}$, led by the charge nurse and the attending physician, with all staff who could attend without compromising patients' safety. A daily huddle form was created by the team to document our compliance to huddle starting time and duration, physician attendance, issues addressed, issue categories (defined to aid later in data analysis), resolution status (resolved or not resolved) and the resolution itself. After every huddle, the paper form is filled out by the charge nurse and placed in a binder.

\section{Huddle log sheet}

To monitor our compliance with the process and track our progress, we developed a huddle log sheet using Excel. The entered data provided information about whether a huddle occurred or not, what issues were addressed, issue categories, resolution and status (closed or open), and when the issue was resolved (during or after the huddle). An automatic report was generated based on the entered time frame.

\section{Implementation strategy}

The huddle was introduced to the multidisciplinary team. A series of plan-do-study-act cycles (PDSAs) was performed to optimise attendance, duration and the sharing of information.

\section{Tool introduction}

Prior to huddle implementation, the concept of the huddle was introduced to the multidisciplinary team in a manner similar to the actual huddle. Staff gathered for a maximum of $15 \mathrm{~min}$ while standing, at the same place and time we intended to conduct our daily huddle. It was explained that a quick report would be given by the charge nurse and the attending physician to share the current status of the unit, the plan for the next 24 hours, 
errors in the past 24 hours, staff concerns and any issue affecting patients or employees. It was made clear that the huddle would be a platform to share knowledge, appreciate teamwork and welcome recommendations. All staff from all disciplines involved in patients' care welcomed the upcoming implementation of the tool.

\section{PDSA 1}

In our PICU, the nursing staff starts their 12 hours shift at 0700 hours, half the nursing staff should start their lunch break at 1200 hours and the second half at 1300 hours. In contrast, physicians start their shift at 0800 hours and start handover to the on-call team at 1530 hours. We thought the best time for a huddle would be 1400 hours. Only a few nurses were able to attend, however, and those who did express that the chosen time was not convenient, because some of them would still be out if they had to start their break late or needed to check on patients immediately afterwards. As a result, the time was changed to 1500 hours, but we soon realised that this left little time to act on some of the issues that were raised. Finally, the huddle time was set at 1430 hours, which improved nurses' attendance and allowed the team to act in a timely manner.

\section{PDSA 2}

Charge nurses left many important elements in the documentation form incomplete, which resulted in missing data. Therefore, multiple sessions with the charge nurses were required to exemplify how to fill out the form as this would ensure complete and accurate documentation. This intervention resulted in immediate improvement.

\section{PDSA 3}

In the same week, we started the daily safety huddle, we realised we needed a mechanism to share and provide feedback on the issues and decisions made during the huddle with team members who could not attend (the majority of the team). Therefore, we started the huddle weekly report, written by a nurse and reviewed by a physician from the huddle team, focusing on the most important issues or topics discussed and emailed to the multidisciplinary team. Positive results came from the weekly report; staff started to refer to solutions and discussions when they encountered similar issues.

\section{PDSA 4}

We tried our best to adhere to a huddle duration of $15 \mathrm{~min}$, but we exceeded that on many occasions. The leading charge nurse, physicians, and staff were reminded to adhere to the time limit and advised to use a timer as an effective reminder.

\section{RESULTS}

We monitored our compliance with the process of the daily safety huddle in the PICU from April to December

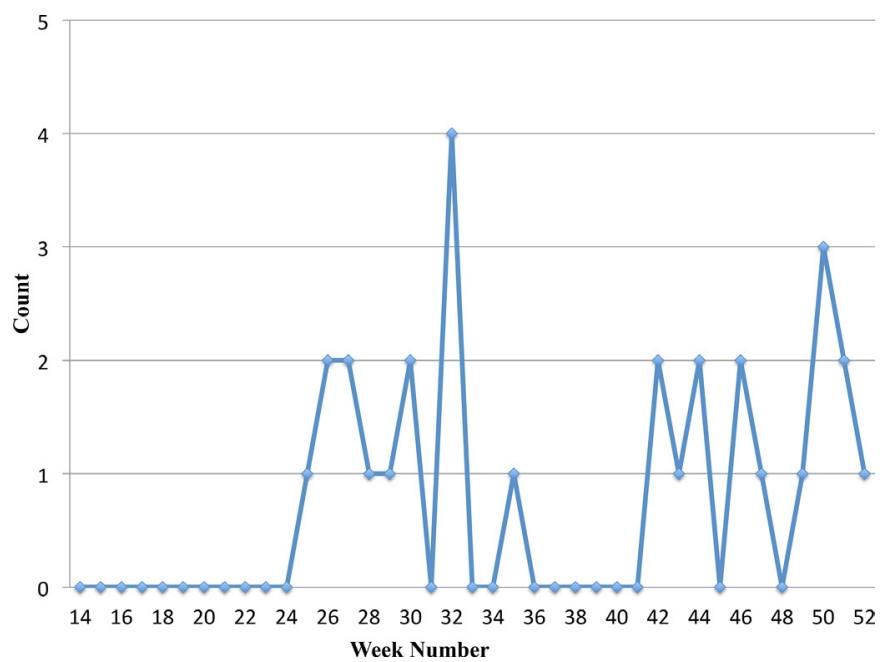

Figure 1 Number of huddles exceeding $15 \mathrm{~min}$. Project started on April which is the week number 14 of the year.

2017. Data were collected and analysed using the huddle $\log$ sheet.

\section{Process measures}

The median start time was 1430 hours, and the number of daily huddles exceeding $15 \mathrm{~min}$ was monitored as well (figure 1).

\section{Outcome measures}

A total of 275 huddles occurred with an initial compliance rate of $73 \%$, which grew up to $97 \%$ (figure 2). We addressed a total of 340 issues, the majority of which were safety issues pertaining to infection control and medication errors $(109 ; 32.05 \%)$, followed by communication $(83 ; 24.41 \%)$, documentation $(59 ; 17.35 \%)$, other issues $(37 ; 10.88 \%)$, procedures $(20 ; 5.88 \%)$, patient flow $(16$; $4.7 \%$ ), and equipment and supplies $(16 ; 4.7 \%)$ (figure 3$)$. Staff perception of safety and teamwork will be re-evaluated in the next hospital culture survey, taking place in 2020.

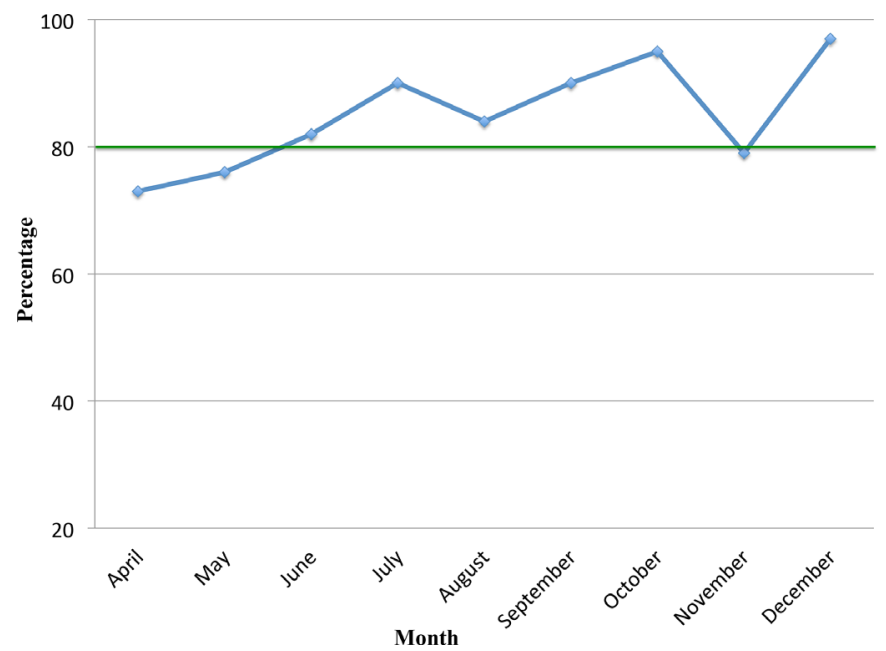

Figure 2 Percentage of monthly huddle compliance. *The green line represents our target. 


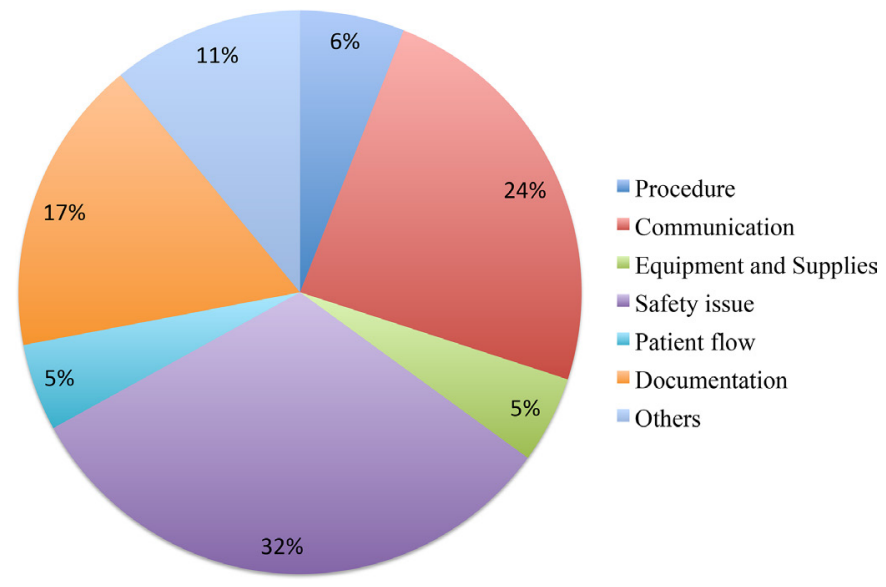

Figure 3 Percentage of issues by category (procedure, communication, equipment and supplies, safety issue, patient flow, documentation and other).

\section{DISCUSSION}

When we started the project, most of the observations made and issues raised came from unit leadership. However, it was a good start for the whole team to meet and discuss workflow and expectations, which helped us understand and appreciate each other's work more. Direct involvement of frontline staff in discussions became easier with time. They started to open up, ask for clarification, discuss concerns and provide suggestions to improve communication between teams; in some instances, they brought up a problem and provided a solution. Most of the issues raised were resolved during the huddle, and a few were resolved afterward. The majority of the unresolved issues were system issues that involved different departments and required escalation to higher management or modification in the hospital information system (figure 4).

When the project was completed and the data were analysed, some categories included subcategories that would have been better separated. Therefore, we used the hospital reporting system as a guide to redefine the categories, focusing on those relevant to our unit. The daily safety huddle can be done in all hospital units, however. Each unit can structure huddles based on the unit's nature and needs. Many staff on rotation in the PICU admired the concept of the daily huddle and the way it

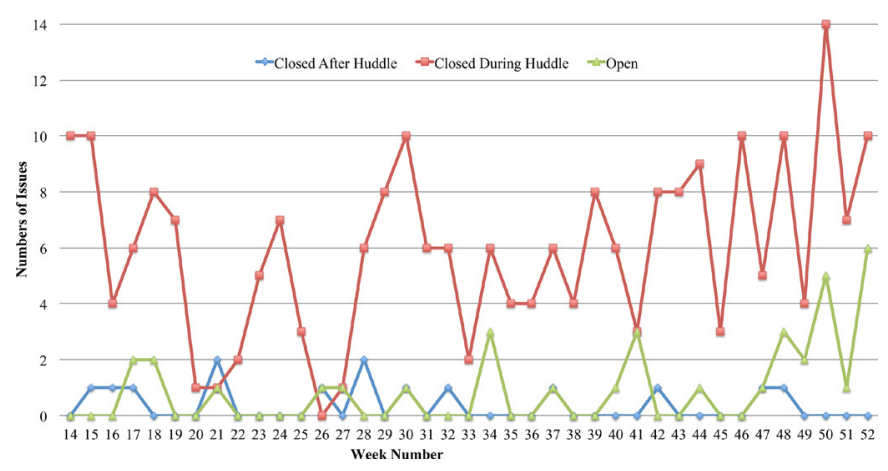

Figure 4 Status of issues raised during huddle (closed after the huddle, closed during the huddle, open). Project started on April which is the week number 14 of the year. brought the teams involved in patients' care together. Moreover, many said that the huddle needed to be done in all hospital units.

The daily safety huddle has become an integral part of the PICU daily routine; the commitment of the unit's leaders and active listening to staff concerns and suggestions keep the huddle going. Every contribution is appreciated; sharing our achievements and explaining how they improve patient care and teamwork brings our unit joy and sustainability. The daily safety huddle accommodated other initiatives to remind the team of the importance of maintaining patients' safety. For instance, we introduced a manual timer updated by staff for zero medication errors and zero unplanned extubation days. Furthermore, some staff started a situational huddle if a situation required immediate attention and role clarification. Looking at our data after project completion, our compliance with the daily huddle remained the same. Moreover, issues unresolved during the huddle which required more discussion with the majority of the team were brought to monthly nursing unit meetings, division meetings or PICU quality team meetings for further discussion.

\section{LESSONS AND LIMITATIONS}

The findings of this study revealed that the medical team was not previously aware of many aspects of nurses' responsibilities in the unit and their workflow. The daily safety huddle diffused a lot of misconceptions and misunderstandings between nursing and medical teams, making work smoother.

In addition, we learnt that providing a daily safety huddle is an effective strategy in reinforcing inconsistencies in practice such as hand hygiene and infection control issues. Delays in entering orders after the multidisciplinary round were addressed in the daily huddle until the issue was resolved. This strategy worked with many major safety issues to ensure staff compliance. Furthermore, the results and data analysis of the daily safety huddle can be used to aid future quality improvement projects. For example, the huddle highlighted the need for an initiative to improve communication between our unit and other departments before and after receiving patients.

Although we aimed to conduct the safety huddle on a daily basis, there were days when it was not possible. Sometimes the time coincided with admissions, procedures, and the majority of staff had to attend to patients. In these cases, the cancellation of the huddle was documented and justification was included. It is important to mention that writing the weekly report was a challenge due to vacations and other commitments. However, this inconsistency was resolved when the project was completed; interested staff joined and volunteered to write the report. To make the process easier, we decided to make the report monthly rather than weekly. 
Entering the data in the log sheet was another challenge we encountered. The log sheet could not be sustained after completion of the project due to work overload.

\section{CONCLUSIONS}

The daily safety huddle helped in early identification and timely resolution of safety issues. Solving issues and providing feedback ameliorated interactions among the multidisciplinary team members and improved subsequent patient care. Sharing knowledge, errors and achievements created trust between frontline staff and leadership. The systematic use of daily safety huddle is a powerful tool to create an equitable environment where frontline staff can speak up freely about daily patient safety concerns. The huddle leads to a more open and active discussion with unit leadership and to the ability to perform the right action at the right time.

Acknowledgements The authors would like to thank all PICU staff for their commitment and contribution to the daily safety huddle, Mr Mohammad Aldawood for his assistance in the project and Editage (www.editage.com) for English language editing.

Contributors AA: approved selection of the tool. FA and HA: planned implementation of the tool. FA: introduced the tool and work flow of the project. YK: supervised the project. FA: took the lead in writing the manuscript. KS: provided critical feedback and suggestions.

Funding The authors have not declared a specific grant for this research from any funding agency in the public, commercial or not-for-profit sectors.

Competing interests None declared.

Patient consent for publication Not required.

Ethics approval Staff confidentiality was maintained for staff who did not agree to discuss errors by themselves.

Provenance and peer review Not commissioned; externally peer reviewed. Data availability statement Data are available upon request.
Open access This is an open access article distributed in accordance with the Creative Commons Attribution Non Commercial (CC BY-NC 4.0) license, which permits others to distribute, remix, adapt, build upon this work non-commercially, and license their derivative works on different terms, provided the original work is properly cited, appropriate credit is given, any changes made indicated, and the use is non-commercial. See: http://creativecommons.org/licenses/by-nc/4.0/.

ORCID iDs

Fatima Aldawood http://orcid.org/0000-0003-1254-6716

Yasser Kazzaz http://orcid.org/0000-0003-3590-4547

\section{REFERENCES}

1 Gooch K. The chronic problem of communication: why it's a patient safety issue, and how hospitals can address it. Becker's Hospital Review, 2016. Available: https://www.beckershospitalreview.com/ quality/the-chronic-problem-of-communication-why-it-s-a-patientsafety-issue-and-how-hospitals-can-address-it.html [Accessed Jan 2020].

2 HFM staff. Daily briefing puts safety first for staff, patients. health facilities management, 2013. Available: https://www.hfmmagazine. com/articles/495-daily-briefing-puts-safety-first-for-staff-patients [Accessed Jan 2020].

3 Institute for Healthcare Improvement. Tools: Huddles. (n.d.). Available: http://www.ihi.org/resources/Pages/Tools/Huddles.aspx [Accessed Jan 2020].

4 NHS Improvement. safety huddles. (n.d.). Available: https:// improvement.nhs.uk/resources/safety-huddles/ [Accessed Jan 2020].

5 Provost SM, Lanham HJ, Leykum LK, et al. Health care huddles: managing complexity to achieve high reliability. Health Care Manage Rev 2015;40:2-12.

6 Eppich WJ, Brannen M, Hunt EA. Team training: implications for emergency and critical care pediatrics. Curr Opin Pediatr 2008;20:255-60.

7 Donnelly LF, Cherian SS, Chua KB, et al. The daily readiness Huddle: a process to rapidly identify issues and foster improvement through problem-solving accountability. Pediatr Radiol 2017;47:22-30.

8 Stapley E, Sharples E, Lachman P, et al. Factors to consider in the introduction of huddles on clinical wards: perceptions of staff on the safe programme. Int J Qual Health Care 2018;30:44-9.

9 King HB, Battles J, Baker DP. Team Strategies and Tools to Enhance Performance and Patient Safety: Performance and Tools. In: Henriksen K, Battles JB, Keyes MA, eds. Advances in patient safety: new directions and alternative approaches. 3rd edn. Rockville (MD): Agency for Healthcare Research and Quality, 2008. 